\title{
Non-Gaussian Stochastic Model for Analysis of Automatic Detection/Recognition
}

\author{
Philip B. Chapple, Derek C. Bertilone, and Steven Angeli \\ Wide Area Surveillance Division \\ Defence Science and Technology Organisation \\ PO Box 1500, Salisbury SA 5108 AUSTRALIA \\ philip.chapple@dsto.defence.gov.au
}

\begin{abstract}
We demonstrate that a very simple stochastic model based on nonlinear transformation of Gaussian random fields can be successfully used to model homogeneous non-Gaussian natural backgrounds observed in a wide range of airborne and spaceborne sensor imagery. We use this model to simulate backgrounds ranging from IR forest terrain to SAR woodland and SAR sea surface imagery. The model reproduces the histogram, second-order correlations, and third-order correlations measured in the real imagery. We discuss applications in the design and analysis of algorithms for automatic detection and recognition of objects embedded in natural imagery.
\end{abstract}

\section{Introduction}

Stochastic models of backgrounds are required for the rigorous analysis of automatic detection and recognition in natural imagery. They are necessary for the design of optimum algorithms; decision theory provides a powerful mathematical framework for designing optimum detection and recognition strategies, but requires knowledge of the probability density for the multidimensional data vector under the assumed hypotheses. Stochastic models are also useful for generating large numbers of background images for Monte Carlo testing of algorithms when the cost of obtaining large quantities of real image data is prohibitive.

A research program is currently underway at DSTO to investigate stochastic modeling approaches to the design and analysis of algorithms for automatic detection and recognition of objects in airborne and spaceborne sensor imagery. A problem is that the spatial statistics of natural backgrounds in high resolution imagery are quite often non-Gaussian ${ }^{1,2}$, and are therefore difficult to characterise and model. The usual approach is to use a stochastic model that is constrained to have a specified histogram and second-order correlation function (but which might differ from the real background in higher-order correlations). Even with this limitation, most techniques currently available are complicated and computationally intensive $e^{3,4}$, and this has restricted their application in detection and recognition problems.

We have found that a very simple stochastic model based on the pointwise nonlinear transformation of Gaussian random fields (GRF's) can be successfully used to model and simulate infrared (IR) homogeneous natural terrain imagery ${ }^{5}$. Our model 
is based on a modification of a method used in Ref. 6, is straightforward to set up and implement, and allows simulations to be generated rapidly.

We show here that the same model can also be successfully applied to synthetic aperture radar (SAR) natural imagery; in particular, airborne SAR woodland and spaceborne SAR sea surface imagery. The woodland imagery is particularly difficult to model and simulate by other means ${ }^{2}$. We have computed histograms, second-order covariances, and some third-order covariances and demonstrate that the model gives results in good agreement with those of the actual data. We describe the current and future directions of our work on applying the model to detection and recognition problems.

\section{The stochastic model}

To set up the model, we pass sample images of the background of interest through an invertible pointwise nonlinear transform that has been specifically designed so that the transformed samples have a Gaussian marginal probability density. The transformation function $F(x)$ is obtained from the histogram $P(x)$ of the original samples:

$$
F(x)=\sqrt{2} \operatorname{erf}^{-1}\left\{2 \int_{0}^{x} P\left(x^{\prime}\right) d x^{\prime}-1\right\} .
$$

Here $\operatorname{erf}^{-1}$ denotes the inverse of the error function. The essence of our model is to treat the transformed samples as realisations of a GRF. We estimate the second-order covariance function for this GRF by directly computing the covariances in the transformed samples. We then simulate the original imagery by generating new realisations of the GRF with this same correlation structure using one of a number of fast algorithms, for example that of Ref. 7, and passing them back through the inverse transformation.

\section{Backgrounds and simulations}

Figure 1 shows three different terrains. On the left are real images obtained from field trials. The IR forest image is from an ensemble of 254 images, each of size $136 \times 272$ pixels, obtained from an airborne sensor in the IR. The SAR woodland image was acquired from an airborne X-band sensor, and is of size $512 \times 512$ pixels. The SAR sea surface image was taken from C-band RADARSAT data (standard mode 3), and is of size $256 \times 256$ pixels. Both SAR images are log-amplitude and have not been preprocessed to reduce speckle.

The figures on the right show simulations using the model. In the case of the IR forest terrain, the model is set up using all of the available images in the ensemble. This is necessary to obtain reliable statistical estimates because of long-range correlations that extend over the width of the imagery ${ }^{1}$. In the case of the SAR images only a single sample image is required. The visual appearance of the simulations is very good, and we have found this to be generally true for backgrounds that are not too highly structured in appearance. 
Real

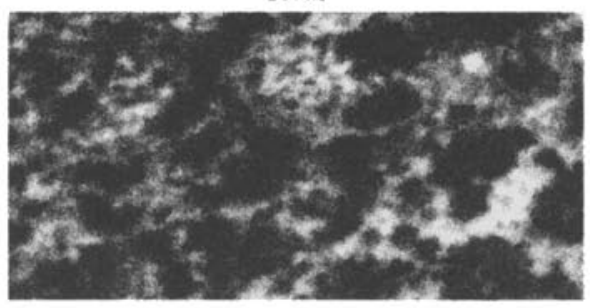

Simulated

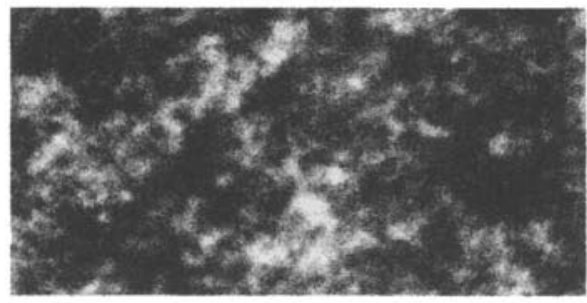

IR image - forest

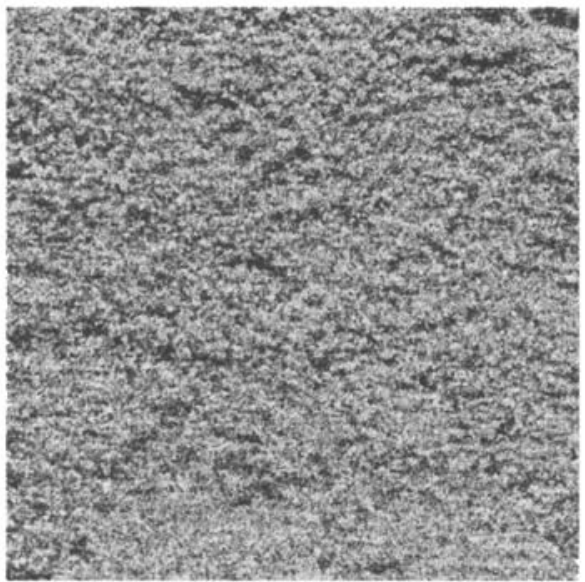

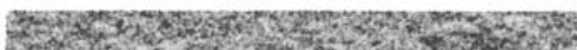
\&3.

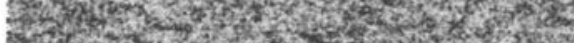

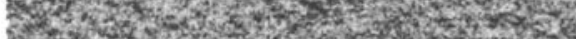

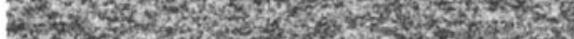

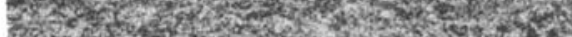

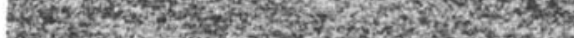

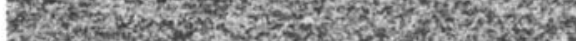

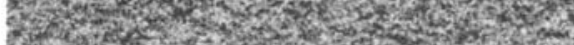

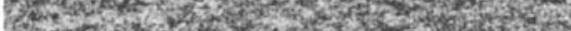

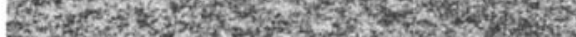

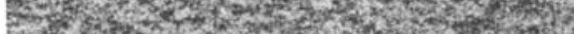

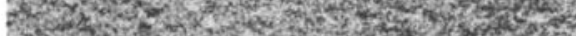

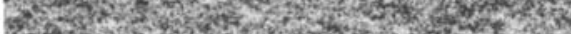
6.0.

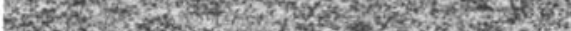

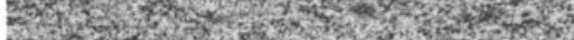

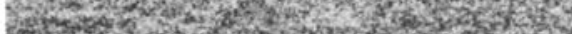

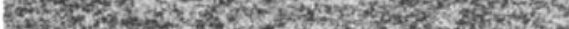

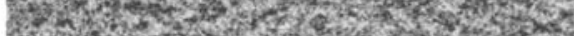

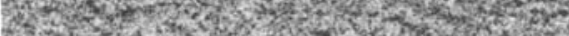

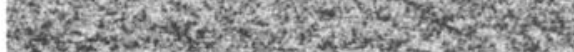

SAR image - woodland
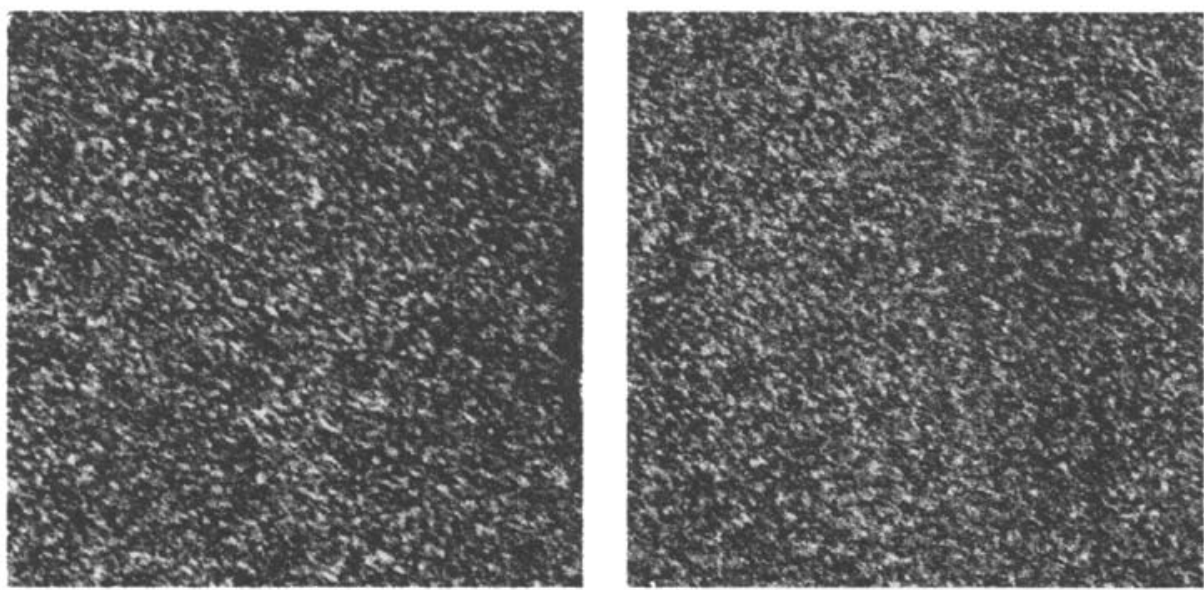

SAR image - sea

Fig. 1. Real and simulated terrain images 


\section{Image statistics}

The simulations reproduce the histograms, second-order covariances, and third-order covariances found in the real imagery, to a good approximation. The second-order and third-order covariances for a homogeneous random field $x(\vec{m})$ with mean $\mu=E[x(\vec{m})]$ are defined as follows:

$$
\begin{gathered}
C_{2}(\vec{k})=E[(x(\vec{m})-\mu)(x(\vec{m}+\vec{k})-\mu)] \\
C_{3}(\vec{k}, \vec{l})=E[(x(\vec{m})-\mu)(x(\vec{m}+\vec{k})-\mu)(x(\vec{m}+\vec{l})-\mu)]
\end{gathered}
$$

Our estimates of these quantities are obtained by spatial averaging over each image in the usual way, and then averaging over the ensemble of images in cases when an ensemble is available (ie. for the IR forest imagery). Figures 2-4 give examples for the IR forest, SAR woodland, and SAR sea surface imagery, respectively. In the case of the IR forest imagery we compute the statistics of the simulations from an ensemble of 254 simulated images, and the 1-standard deviation error-bars shown are obtained in the manner described in Ref. 1.

In each of these figures, (a) shows the histograms for real and simulated imagery, (b) shows the second-order covariance in the horizontal and vertical directions for real (dash-dot) and simulated (solid) imagery, and (c) shows some third-order covariances for real (dash-dot) and simulated (solid) imagery. The third-order covariances correspond to pixel displacements $\vec{k}=\overrightarrow{0}$ and $\vec{l}$ lying in the horizontal and vertical directions.

The IR forest and SAR woodland backgrounds exhibit rich correlation structures that extend over many pixels, while the SAR sea surface image exhibits correlations over a small number of pixels. Although the model does not explicitly constrain the covariances to match those in the real background, the second-order and third-order covariances appear to be in good agreement; in cases where we have enough data for reasonable error-bar estimates, the real and simulated covariances lie within the errorbars for much of the lag range (see Fig. 2).

\section{Current and future directions: automatic detection/recognition}

Our current investigations involve the analysis of automatic detection and recognition in airborne and spaceborne sensor imagery. We have evaluated the effectiveness of the model for Monte Carlo testing of small target detection algorithms and have obtained promising results. We have inserted artificial targets into ensembles of real and simulated imagery, passed them through a number of detection algorithms and computed probabilities of detection and false alarm rates. Figure 5 shows a typical result for point target detection using a $3 \times 3$ median subtraction filter followed by a threshold. The results for a simple global threshold are also shown for comparison. Statistical performance predictions obtained using the model are in good agreement with those using real imagery. We are currently evaluating the model against more complex algorithms for extended target recognition. 

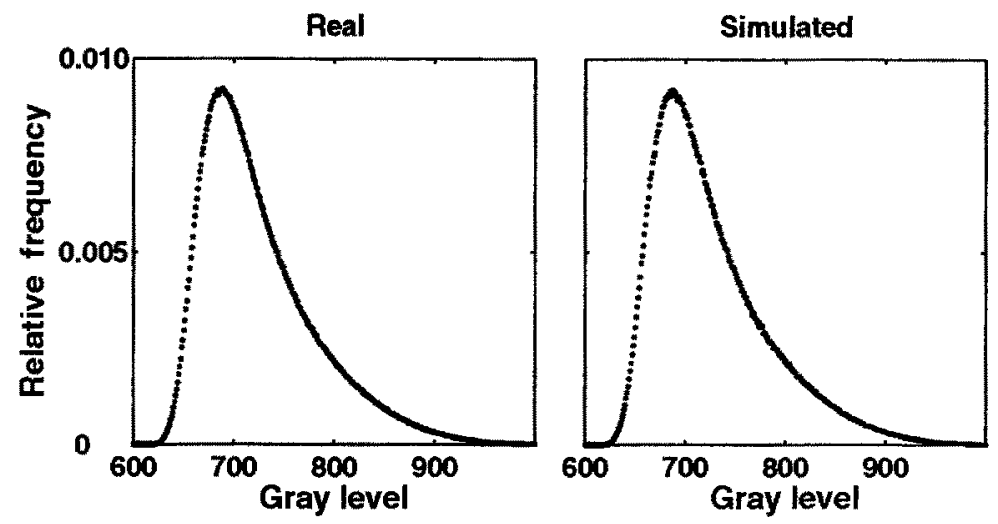

(a) Histograms
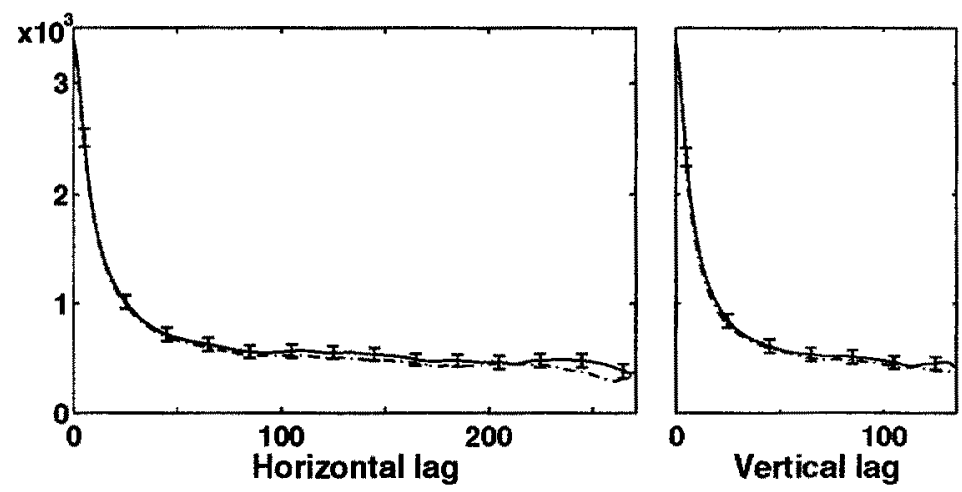

(b) Second-order covariance estimates: ... real; - - simulated
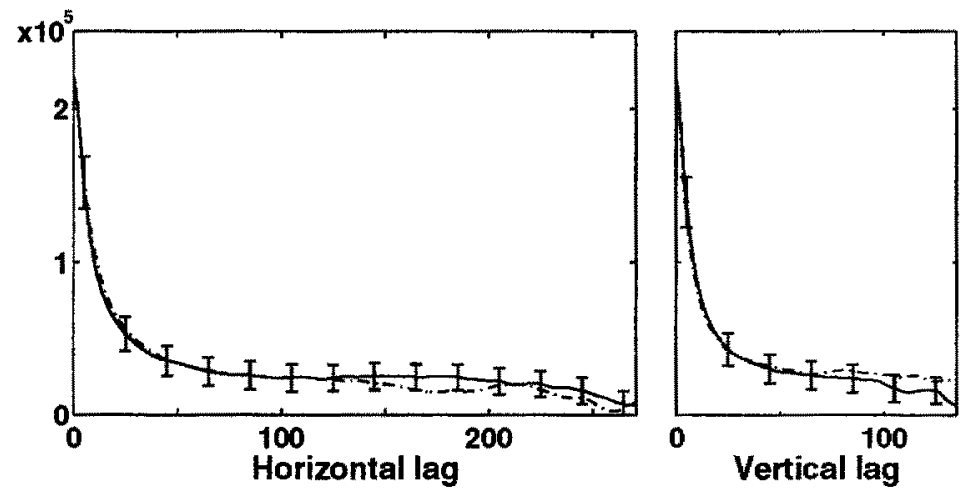

(c) Third-order covariance estimates: $\quad \cdots$ real; - simulated

Fig. 2. Statistics of IR forest image ensemble 


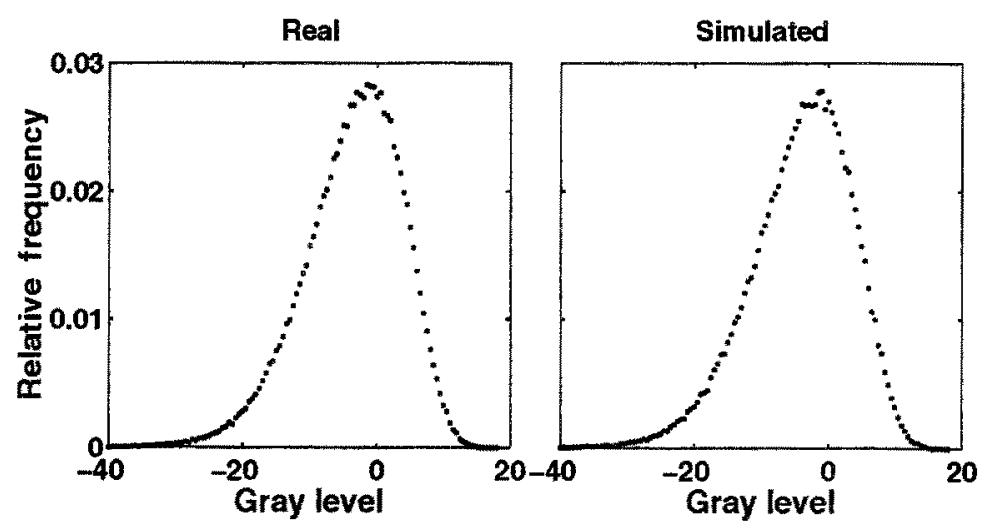

(a) Histograms
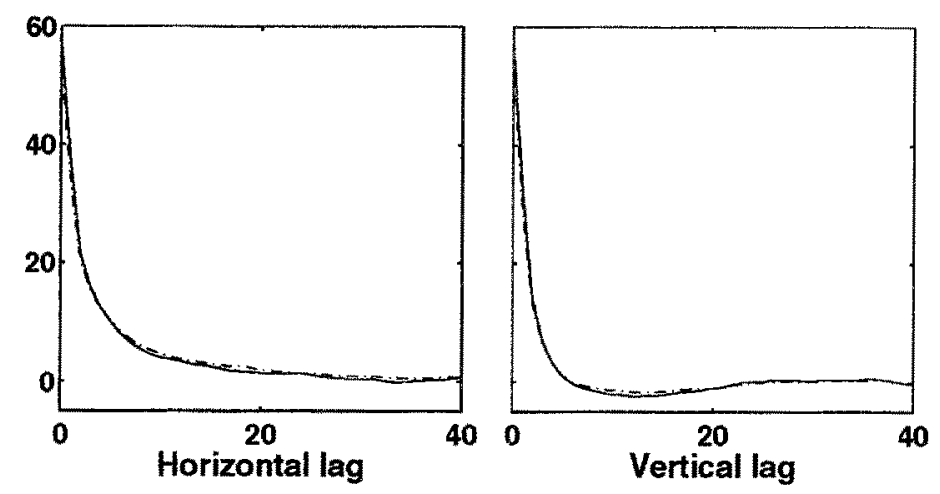

(b) Second-order covariance estimates: $\quad$-.. real; - - simulated
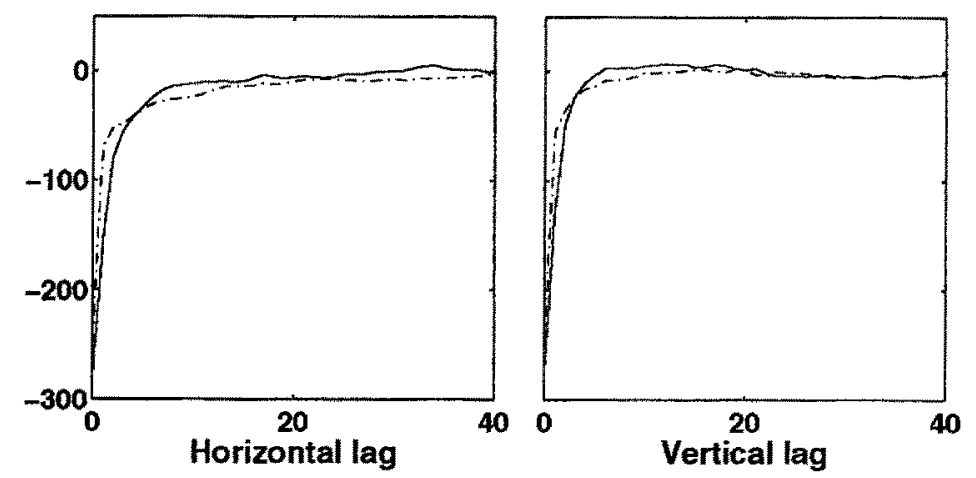

(c) Third-order covariance estimates: ... real; - - simulated

Figure 3. Statistics of SAR woodland image 

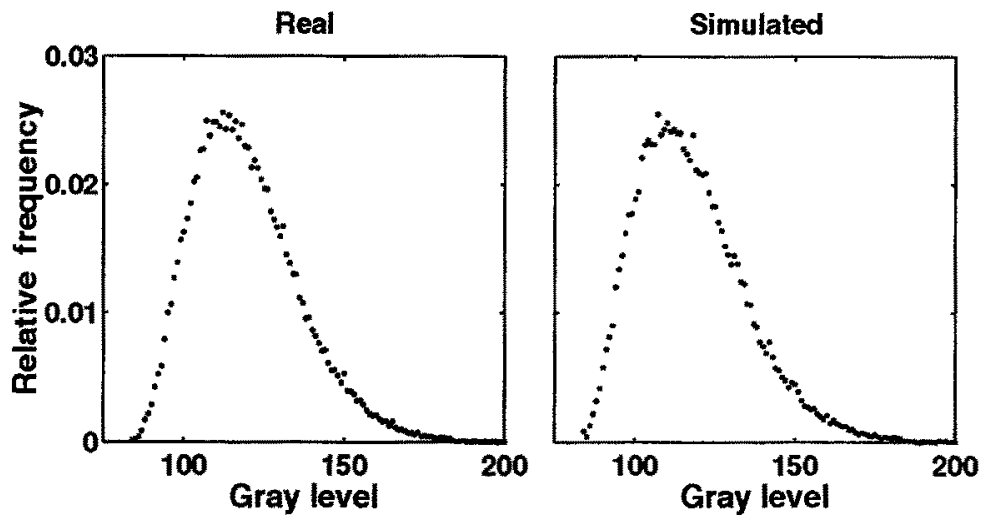

(a) Histograms
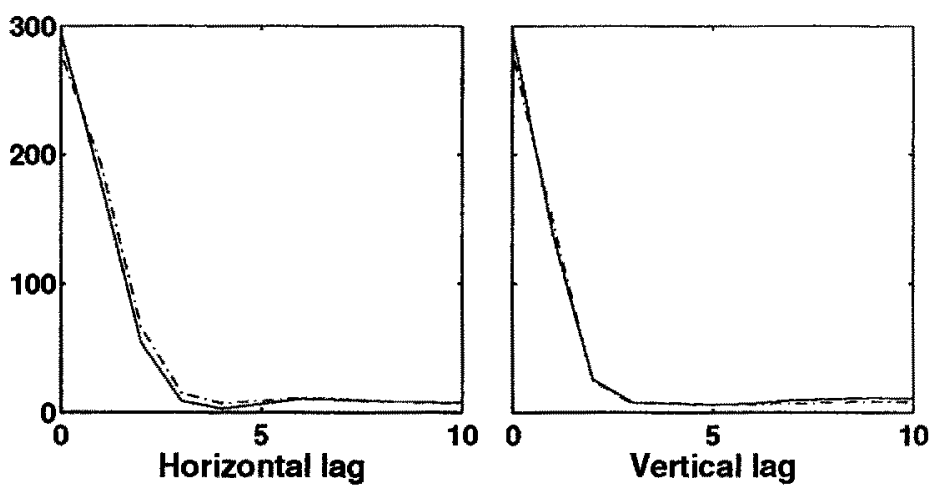

(b) Second-order covariance estimates: $\cdots$ real; - simulated
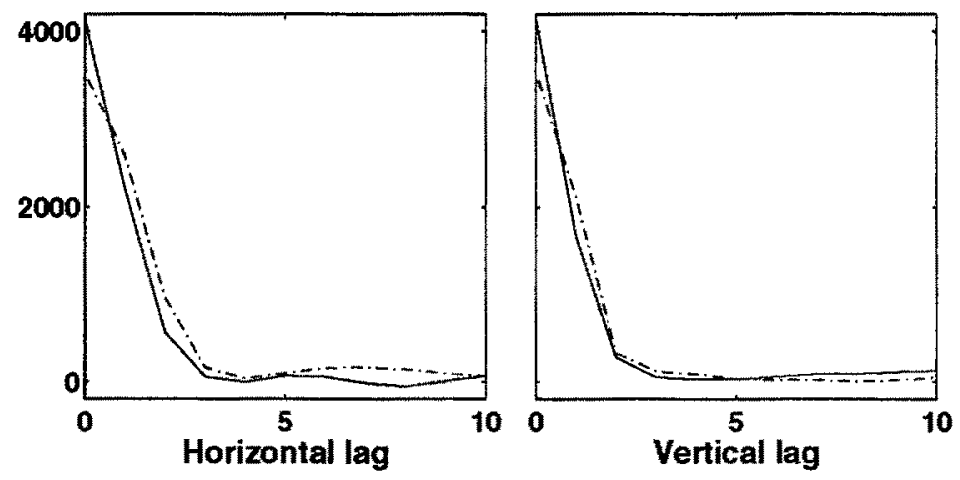

(c) Third-order covariance estimates: ... real; - - simulated

Figure 4. Statistics of SAR sea image 


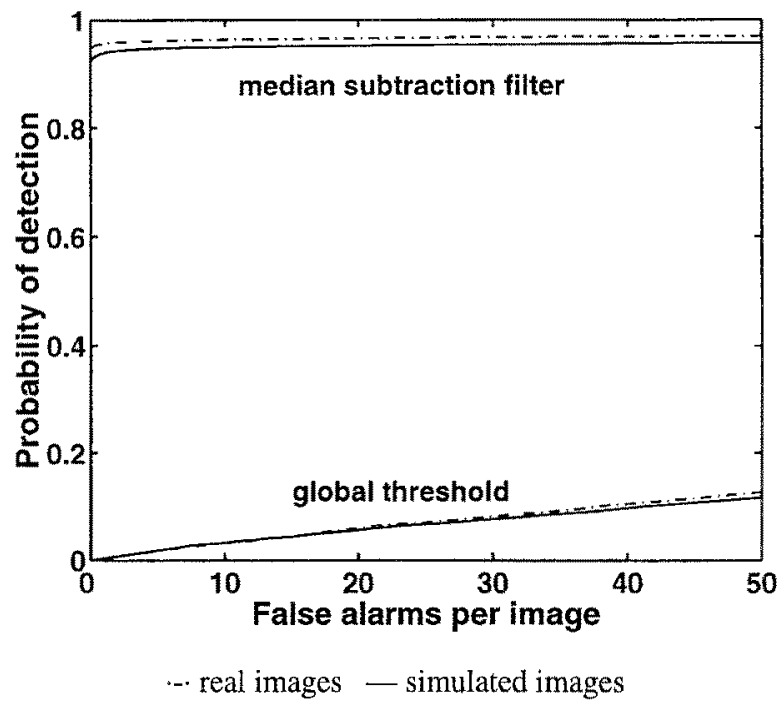

Figure 5. Receiver operating characteristics for detection of small targets inserted in IR forest images

Future work will involve the design of optimum algorithms. One promising approach is to transform the imagery into the "Gaussian-space" where the probability density for the multidimensional data vector for the background can be taken to be known, and then designing an optimum algorithm for the transformed imagery. Since the transform is invertible, no information is lost in the process.

\section{Acknowledgments}

We thank Garry Newsam for numerous helpful discussions. We also thank Imaging Surveillance Systems group of Tactical Surveillance Systems Division and Surveillance Systems Assessment group of Wide Area Surveillance Division for providing the SAR image data.

\section{References}

1. Bertilone, R.S. Caprari, S. Angeli and G.N. Newsam, Applied Optics 36 (1997) 9167

2. M.L. Williams, S. Quegan and D. Blacknell, Waves in Random Media 7 (1997) 643

3. G.E. Johnson, Proceedings of the IEEE 82 (1994) 270

4. V.V. Tatarskii and V.I. Tatarskii, Waves in Random Media 6 (1996) 419

5. P.B. Chapple and D.C. Bertilone, "Stochastic simulation of infrared non-Gaussian natural terrain imagery," to appear in Optics Communications (1998)

6. G.N.Newsam and M.Wegener, "Generating non-Gaussian random fields for sea surface simulations," Proceedings 1994 ICASSP, April 19-22, Adelaide, South Australia

7. C.R.Dietrich and G.N.Newsam, Water Resources Research 29 (1993) 2861 